\title{
An Alternative Criterion to Likelihood for Parameter Estimation Accounting for Prior Information on Nuisance Parameter
}

Adel Mohammadpour ${ }^{1}$ and Ali Mohammad-Djafari ${ }^{2}$

1 Permanent address: Department of Statistics, Faculty of Mathematics \& Computer Science, Amirkabir University of Technology, 424 Hafez Ave., 15914 Tehran, and School of intelligent Systems, IPM, Iran, adel@aut.ac.ir

$2{ }^{1}$ Laboratoire des Signaux et Systèmes, Unité mixte de recherche 8506 (CNRS-Supélec-UPS) Supélec, Plateau de Moulon, 91192 Gif-sur-Yvette, France \{mohammadpour, djafari\}@lss.supelec.fr

In this paper we propose an alternative tool to marginal likelihood for parameter estimation when we want to account for some prior information on a nuisance parameter. This new criterion is obtained using the median in place of the mean when using a prior distribution on the nuisance parameter. We first give the precise definition of this new criterion and its properties and then present a few examples to show their differences.

\section{Introduction}

Assume that we are given an observation $x$ with cumulative distribution function (cdf) $F_{X \mid \mathcal{V}, \theta}(x \mid \nu, \theta)$ (or probability density function (pdf) $f_{X \mid \mathcal{V}, \theta}(x \mid \nu, \theta)$ ) with two unknown parameters $\nu$ and $\theta$. We assume that $\nu$ is a nuisance parameter on which we have an a priori information translated by a prior distribution $F_{\mathcal{V}}(\nu)$ (or a pdf $f_{\mathcal{V}}(\nu)$ ) and we want to infer on $\theta$.

If $\nu$ was given, i.e. $\nu=\nu_{0}$, then the classical Maximum Likelihood (ML) estimate of $\theta$ is defined as the optimizer of the likelihood function

$$
l(\theta)=f_{X \mid \nu, \theta}\left(x \mid \nu_{0}, \theta\right) .
$$

The question is now how to account for the prior $F_{\mathcal{V}}(\nu)$. Again the classical solution is to integrate out $\nu$ to obtain the marginal pdf

$$
f_{X \mid \theta}(x \mid \theta)=\int f_{X \mid \mathcal{V}, \theta}(x \mid \nu, \theta) f_{\mathcal{V}}(\nu) \mathrm{d} \nu
$$

and then estimate $\theta$ by optimizing the likelihood function 


$$
l(\theta)=f_{X \mid \theta}(x \mid \theta) .
$$

In this work, we propose a new inference tool $\widetilde{F}_{X \mid \theta}(x \mid \theta)$ which can be used to do the same inference on $\theta$. This new inference tool is deduced from the interpretation of $F_{X \mid \theta}(x \mid \theta)$ as the mean value $F_{X \mid \mathcal{V}, \theta}(x \mid \nu, \theta)$ using the pdf of $f_{\mathcal{V}}(\nu)$. Now, if in place of the mean value we take the median we obtain this new inference tool $\widetilde{F}_{X \mid \theta}(x \mid \theta)$ which is defined as

$$
\widetilde{F}_{X \mid \theta}(x \mid \theta): \mathrm{P}\left(F_{X \mid \mathcal{V}, \theta}(x \mid \mathcal{V}, \theta) \leq \widetilde{F}_{X \mid \theta}(x \mid \theta)\right)=1 / 2
$$

and can be used in the same way to estimate $\theta$ by optimizing

$$
\widetilde{l}(\theta)=\widetilde{f}_{X \mid \theta}(x \mid \theta),
$$

where $\widetilde{f}_{X \mid \theta}(x \mid \theta)$ is the pdf corresponding to the cdf $\widetilde{F}_{X \mid \theta}(x \mid \theta)$.

As far as the authors know, this alternative tool is newly presented [1, $2]$ and applied for hypothesis testing. In this paper we consider its use for parameter estimation.

In the following, first we give more precise definition of $\widetilde{F}_{X \mid \theta}(x \mid \theta)$. Then we present some of its properties, for example, we show that under some conditions $\widetilde{F}_{X \mid \theta}(x \mid \theta)$ has all the properties of a cdf, its calculation is very simple and is robust relative to the prior distribution. Then, we give a few examples and finally, we compare the relative performances of these two tools for estimating of $\theta$.

\section{A New Inference Tool}

Hereafter in this section to simplify the notations we omit the parameter $\theta$.

Definition 1. Let $X$ have a cdf depending on random parameter $\mathcal{V}$ with pdf $f_{\mathcal{V}}(\nu)$. The marginal cdf of $X$ based on median, $\widetilde{F}_{X}(x)$, is defined as the median of $F_{X \mid \mathcal{V}}(x \mid \nu)$ over $f_{\mathcal{V}}(\nu)$.

To simplify calculations of $\widetilde{F}_{X}(x)$, we use definition of median in statistics. That is we calculate $\widetilde{F}_{X}(x)$ by solving the following equation

$$
F_{F_{X \mid \mathcal{V}}(x \mid \mathcal{V})}\left(\widetilde{F}_{X}(x)\right)=\frac{1}{2}, \quad \text { or equivalently } \quad P\left(F_{X \mid \mathcal{V}}(x \mid \mathcal{V}) \leq \widetilde{F}_{X}(x)\right)=\frac{1}{2} .
$$

Theorem 1. Let $\widetilde{F}_{X}(x)$ be the function defined in (1).

1. $\widetilde{F}_{X}(x)$ is a non-decreasing function.

2. If $F_{X \mid \mathcal{V}}(x \mid \nu)$ and $F_{\mathcal{V}}(\nu)$ are continuous cdfs and the random variable $T=F_{X \mid \mathcal{V}}(x \mid \mathcal{V})$ has an increasing cdf (for all fixed $x$ ) then $\widetilde{F}_{X}(x)$ is a continuous function. 
3. $0 \leq \widetilde{F}_{X}(x) \leq 1$.

Proof: 1. Let $x_{1}<x_{2}$. For $i=1,2$, take

$$
k_{i}=\widetilde{F}_{X_{i}}\left(x_{i}\right) \quad \text { and } \quad Y_{i}=F_{X_{i} \mid \mathcal{V}}\left(x_{i} \mid \nu\right) .
$$

Then using 1 we have

$$
P\left(Y_{1} \leq k_{1}\right)=P\left(Y_{2} \leq k_{2}\right)=\frac{1}{2} .
$$

We also have

$$
Y_{1} \leq Y_{2}
$$

Therefore,

$$
P\left(Y_{1} \leq k_{1}\right)=P\left(Y_{2} \leq k_{2}\right) \leq P\left(Y_{1} \leq k_{2}\right),
$$

i.e. $k_{1} \leq k_{2}$ or equivalently $\widetilde{F}_{X}(x)$ is non-decreasing.

2. If $\widetilde{F}_{X}(x)$ is a non-decreasing function, then

$$
\widetilde{F}_{X}\left(x_{-}\right)=\lim _{t \uparrow x} \widetilde{F}_{X}(t) \text { and } \tilde{F}_{X}\left(x_{+}\right)=\lim _{t \downarrow x} \widetilde{F}_{X}(t)
$$

exist and are finite (e.g. [3]).

Further, $F_{X \mid \mathcal{V}}(x \mid \nu)$ is continuous with respect to $x$, and so

$$
\begin{aligned}
& P\left(F_{X \mid \nu}\left(x_{-} \mid \nu\right) \leq \widetilde{F}_{X}\left(x_{-}\right)\right)=P\left(F_{X \mid \mathcal{V}}(x \mid \nu) \leq \widetilde{F}_{X}\left(x_{-}\right)\right), \\
& P\left(F_{X \mid \nu}\left(x_{+} \mid \nu\right) \leq \widetilde{F}_{X}\left(x_{+}\right)\right)=P\left(F_{X \mid \mathcal{V}}(x \mid \nu) \leq \widetilde{F}_{X}\left(x_{+}\right)\right) .
\end{aligned}
$$

And by (1) we have

$$
\begin{aligned}
P\left(F_{X \mid \mathcal{V}}(x \mid \nu) \leq \widetilde{F}_{X}\left(x_{-}\right)\right) & =P\left(F_{X \mid \mathcal{V}}(x \mid \nu) \leq \widetilde{F}_{X}(x)\right) \\
& =P\left(F_{X \mid \mathcal{V}}(x \mid \nu) \leq \widetilde{F}_{X}\left(x_{+}\right)\right) .
\end{aligned}
$$

If $Y=F_{X \mid \mathcal{V}}(x \mid \nu)$ has an increasing distribution function, then

$$
\widetilde{F}_{X}\left(x_{-}\right)=\widetilde{F}_{X}(x)=\widetilde{F}_{X}\left(x_{+}\right)
$$

and by $(2) \widetilde{F}_{X}(x)$ is continuous.

3. On the other hand $\widetilde{F}_{X}(x)$ is the median of $Y, 0 \leq Y \leq 1$, and so

$$
0 \leq \widetilde{F}_{X}(x) \leq 1 .
$$

Remark 1 By part 1 of Theorem $1, \lim _{x \uparrow+\infty} \widetilde{F}_{X}(x)$ and $\lim _{x \downarrow-\infty} \widetilde{F}_{X}(x)$ exist. Therefore $\widetilde{F}_{X}(x)$ is a continuous cdf if conditions of Theorem 1 hold and $\lim _{x \downarrow-\infty} \widetilde{F}_{X}(x)=0, \lim _{x \uparrow \infty} \widetilde{F}_{X}(x)=1$. 
Theorem 2. Let $F_{X \mid \mathcal{V}}(x \mid \nu)$ and $F_{\mathcal{V}}(\nu)$ be continuous cdfs. If $L(\nu)=F_{X \mid \mathcal{V}}(x \mid \nu)$ is a monotone function with respect to $\nu$, then $\widetilde{F}_{X}(x)=L\left(F_{\mathcal{V}}^{-1}\left(\frac{1}{2}\right)\right)$.

Proof By (1) we have,

$$
\begin{aligned}
& P\left(L(\mathcal{V}) \leq \widetilde{F}_{X}(x)\right)=\frac{1}{2} \\
\Leftrightarrow & \left\{\begin{array}{l}
P\left(\mathcal{V} \leq L^{-1}\left(\widetilde{F}_{X}(x)\right)\right)=\frac{1}{2} \text { if } L \text { is an increasing function } \\
P\left(\mathcal{V} \geq L^{-1}\left(\widetilde{F}_{X}(x)\right)\right)=\frac{1}{2} \text { if } L \text { is a decreasing function }
\end{array}\right. \\
\Leftrightarrow & \left\{\begin{array}{c}
F_{\mathcal{V}}\left(L^{-1}\left(\widetilde{F}_{X}(x)\right)\right)=\frac{1}{2} \quad \text { if } L \text { is an increasing function } \\
1-F_{\mathcal{V}}\left(L^{-1}\left(\widetilde{F}_{X}(x)\right)\right)=\frac{1}{2} \text { if } L \text { is a decreasing function }
\end{array}\right. \\
\Leftrightarrow & F_{\mathcal{V}}\left(L^{-1}\left(\widetilde{F}_{X}(x)\right)\right)=\frac{1}{2} \\
\Leftrightarrow & \widetilde{F}_{X}(x)=L\left(F_{\mathcal{V}}^{-1}\left(\frac{1}{2}\right)\right),
\end{aligned}
$$

where the last inequality follows from non-decreasing property of the cdf of $\mathcal{V}$.

Remark 2 Let conditions of Theorem 2 be hold. If $\widetilde{F}_{X}(x)$ is a cdf then $\widetilde{F}_{X}(x)$ belong to the family of distribution $F_{X \mid \mathcal{V}}(x \mid \nu)$, because $\widetilde{F}_{X}(x)=$ $F_{X \mid \mathcal{V}}\left(x \mid F_{\mathcal{V}}^{-1}\left(\frac{1}{2}\right)\right)$.

Remark $3 \widetilde{F}_{X}(x)$ is depend on the median of prior distribution, $F_{\mathcal{V}}^{-1}\left(\frac{1}{2}\right)$, (but for calculating the marginal distribution of $X$ we need the prior). Therefore $\widetilde{F}_{X}(x)$ is robust relative to prior distributions with the same medians.

In the following two theorems we show that some important families of cdfs have a monotone distribution function with respect to their parameters and so, calculating of $\widetilde{F}_{X}(x)$ is very easy by using Theorem 2 .

Theorem 3. Let $L(\nu)=F_{X \mid \mathcal{V}}(x \mid \nu)$ and $F_{X \mid \mathcal{V}}(x \mid \nu)$ be an increasing function with respect to $x$. If $\nu$ is a location (scale) parameter then $L(\nu)$ is decreasing (monotone) with respect to $\nu$.

Theorem 4. Let $X \mid \nu$ be distributed according to an exponential family with $p d f$

$$
f_{X \mid \mathcal{V}}(x \mid \nu)=h(x) \exp (\nu T(x)-A(\nu)),
$$

where $T$ and $A$ are real functions. Then $L(\nu)=F_{X \mid \mathcal{V}}(x \mid \nu)$ is a monotone function with respect $\nu$.

\section{Examples}

In this section we give a few simple examples to illustrate properties of the new inference tool. First we consider the exponential distribution with the following distribution function 
$F_{X \mid \mathcal{V}, \theta}(x \mid \nu, \theta)=1-\exp (-\nu(x-\theta)), x>\theta, \theta>0, f_{\mathcal{V}}(\nu)=\exp (-\nu), \nu>0$,

where $1 / \nu$ is the scale parameter. We can use Theorem 3 for calculating $\widetilde{F}_{X \mid \theta}(x \mid \theta)$. By noting that, the median of $\mathcal{V}$ is $\ln 2$, we have

$$
\widetilde{F}_{X \mid \theta}(x \mid \theta)=1-\exp (-\ln 2(x-\theta)), x>\theta, \theta>0 .
$$

On the other hand we can calculate $F_{X \mid \theta}(x \mid \theta)$ by integrating over $f_{\mathcal{V}}(\nu)$ i.e.

$$
F_{X \mid \theta}(x \mid \theta)=1-\frac{1}{(x-\theta)+1}, \quad x>\theta, \theta>0 .
$$

Note that in this problem MLE based on $\widetilde{l}(\theta)=\widetilde{f}_{X \mid \theta}(x \mid \theta)$ and $l(\theta)=f_{X \mid \theta}(x \mid \theta)$ are the same.

The second example is the normal distribution with pdf

$$
f_{X \mid \mathcal{V}, \theta}(x \mid \nu, \theta)=\frac{1}{\sqrt{2 \pi \theta}} \exp \left(-\frac{1}{2}\left(\frac{x-\nu}{\sqrt{\theta}}\right)^{2}\right), \theta>0,
$$

where $\mathcal{V}$ has a standard normal distribution. In this case the median of $\mathcal{V}$ is zero and so (by using Theorem 3 or 4),

$$
\widetilde{f}_{X \mid \theta}(x \mid \theta)=\frac{1}{\sqrt{2 \pi \theta}} \exp \left(\frac{-x^{2}}{2 \theta}\right), \theta>0,
$$

and also we can calculate

$$
f_{X \mid \theta}(x \mid \theta)=\frac{1}{\sqrt{2 \pi(\theta+1)}} \exp \left(\frac{-x^{2}}{2(\theta+1)}\right), \theta>0 .
$$

The MLE based on $\widetilde{l}(\theta)$ is $X^{2}$ and base on $l(\theta)$ is $\max \left(X^{2}-1,0\right)$.

Figure 1 shows the estimated mean absolute error, $E_{X \mid \theta}(|\mathrm{MLE}-\theta|)$, of these estimators, computed using 200000 samples generated from $X \mid \mathcal{V}, \theta \sim$ $N(\mathcal{V}, \theta)$ and $\mathcal{V} \sim N(0,1)$ for $\theta=[0.01: 0.1: 10]$.

\section{Conclusion}

We introduced an alternative inference tool $\widetilde{l}(\theta)$ to the marginal likelihood $l(\theta)$ for using prior information by defining a marginal function $\widetilde{F}_{X \mid \theta}(x \mid \theta)$ which is based on median in place of $F_{X \mid \theta}(x \mid \theta)$ which is the expected value of $\widetilde{F}_{X \mid \theta}(x \mid \theta)$ with respect to $f_{\mathcal{V}}(\nu)$. We proved that, based on a few conditions, $\widetilde{F}_{X \mid \theta}(x \mid \theta)$ is a cdf. Indeed, the computation of $\widetilde{l}(\theta)$ is easier than $l(\theta)$ for two important classes of distributions which are exponential and location-scale family of distributions. $\widetilde{l}(\theta)$ depends only on the median of $f_{\mathcal{V}}(\nu)$ and thus, it is robust with respect to prior distributions with the same median. 


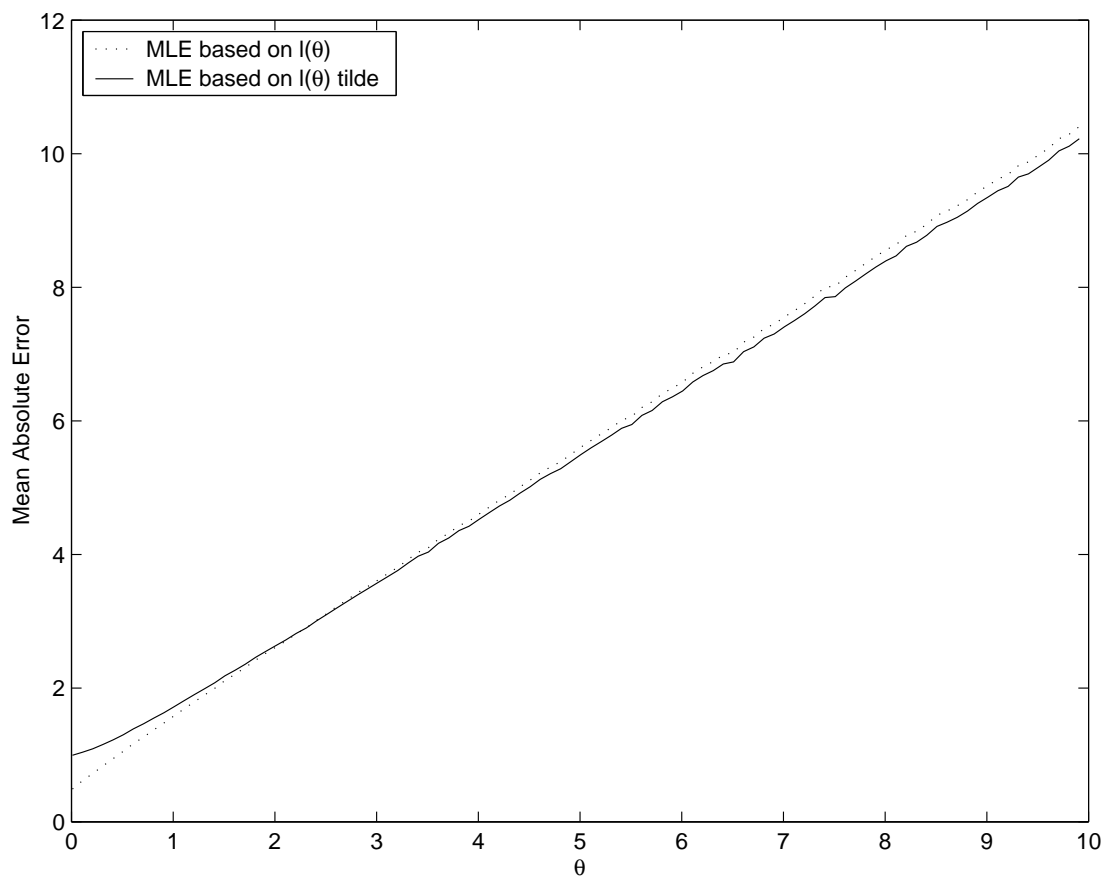

Fig. 1. Mean absolute errors for MLE based on $\widetilde{l}(\theta)$ and $l(\theta)$ i.e. $X^{2}$ and $\max \left(X^{2}-\right.$ $1,0)$, respectively.

\section{References}

1. Mohammadpour A. (2003). Fuzzy Parameter and Its Application in Hypothesis Testing, Technical Report, School of Intelligent Systems, IPM, Tehran, Iran.

2. Mohammadpour A. and Mohammad-Djafari A. (2004). An alternative inference tool to total probability formula and its applications 2003, August, to appear in Proceeding of MAXENT23.

3. Rohatgi V.K. (1976). An Introduction to Probability Theory and Mathematical Statistics. John Wiley and Sons, New York. 